
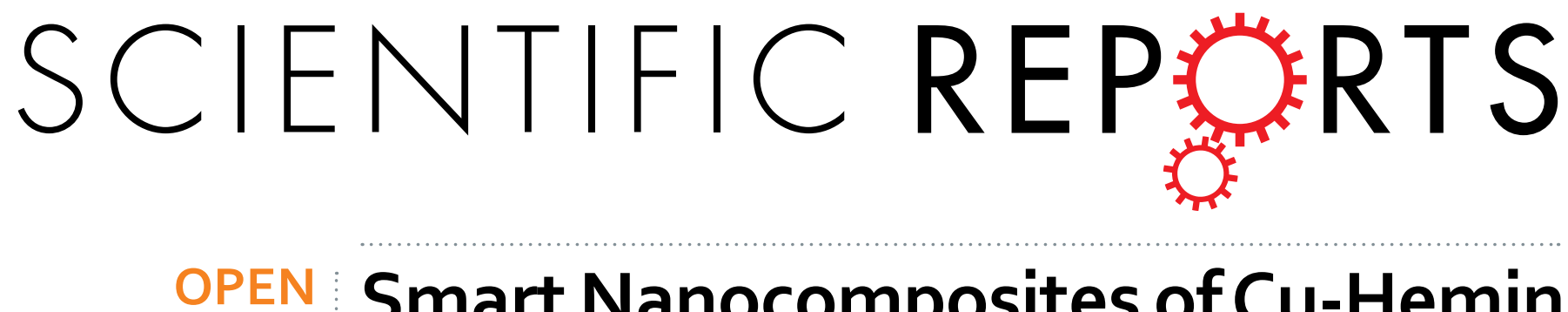

\title{
Smart Nanocomposites of Cu-Hemin Metal-Organic Frameworks for Electrochemical Glucose Biosensing
}

Received: 16 August 2016

Accepted: 14 October 2016

Published: 04 November 2016

\section{Juan He, Han Yang, Yayun Zhang, Jie Yu, Longfei Miao, Yonghai Song \& Li Wang}

Herein, a smart porous material, Cu-hemin metal-organic-frameworks (Cu-hemin MOFs), was synthesized via assembling of $\mathrm{Cu}^{2+}$ with hemin to load glucose oxidase (GOD) for electrochemical glucose biosensing for the first time. The formation of the Cu-hemin MOFs was verified by scanning electron microscopy, $\mathrm{X}$-ray powder diffraction, Fourier transform infrared spectroscopy, $\mathrm{N}_{2}$ adsorption/ desorption isotherms, UV-vis absorption spectroscopy, fluorescence spectroscopy, thermal analysis and electrochemical techniques. The results indicated that the Cu-hemin MOFs showed a ball-flowerlike hollow cage structure with a large specific surface area and a large number of mesopores. A large number of GOD molecules could be successfully loaded in the pores of Cu-hemin MOFs to keep their bioactivity just like in a solution. The GOD/Cu-hemin MOFs exhibited both good performance toward oxygen reduction reaction via Cu-hemin MOFs and catalytic oxidation of glucose via GOD, superior to other GOD/MOFs and GOD/nanomaterials. Accordingly, the performance of GOD/Cu-hemin MOFs-based electrochemical glucose sensor was enhanced greatly, showing a wide linear range from $9.10 \mu \mathrm{M}$ to $36.0 \mathrm{mM}$ and a low detection limit of $2.73 \mu \mathrm{M}$. Moreover, the sensor showed satisfactory results in detection of glucose in human serum. This work provides a practical design of new electrochemical sensing platform based on MOFs and biomolecules.

As an important research area of analytical chemistry ${ }^{1-5}$, electrochemical glucose biosensors have received significant attention over past few years because of their low cost, quick response, simple preparation and wide applications in biomedical, clinical research, food production, ecology and even textile industry ${ }^{6-9}$. Particularly, glucose oxidase (GOD)-based glucose biosensors have been one of the hot spots in analytical chemistry as the introduction of nanomaterials ${ }^{10,11}$.

Various nanomaterials (such as graphene ${ }^{12}$, carbon nanotubes ${ }^{13}$, metal or metal oxide nanostructures ${ }^{14}$ as well as their nanocomposites) have been extensively employed to immobilize GOD on electrode surface for developing electrochemical glucose biosensors owing to their high specific surface area, fast electron transfer, good affinity for enzymes/proteins, excellent catalytic activity ${ }^{15}$ and remarkable biocompatibility ${ }^{7,16,17}$. As previously reported, nanomaterials could both improve the direct electron transfer between the eletroactive center of enzymes/proteins and the electrode surface, and load enzymes/proteins on the electrode surface effectively ${ }^{18-22}$. However, nanomaterials always form close-packed structures after they are assembled on electrode surface, which hinders their performance greatly ${ }^{23-28}$. Furthermore, most of GOD-based biosensors detect glucose level via monitoring the consumption of $\mathrm{O}_{2}$ in enzymatic reaction ${ }^{29}$. So, the nanomaterials are used not only as a supporting to load GOD molecules but also as a catalyst for the reduction of $\mathrm{O}_{2}$ in which the amount of $\mathrm{O}_{2}$ consumed in the enzymatic reaction could be explored ${ }^{19,30,31}$. Paradoxically, excess GOD immobilized on nanomaterials could enhance the performance of GOD, yet hinder the reduction of $\mathrm{O}_{2}$, which still results in a poor performance. Thus, there are still challenges to develop new materials for constructing GOD-based glucose biosensors.

Metal-organic frameworks (MOFs) and zeolitic imidazolate frameworks (ZIFs) own perfect physical and chemical properties such as three-dimensional (3D) structure, high specific surface area, multiple inner porosity and high crystalline. They have been extensively applied in gas storage and separation, drug delivery, catalysis, clinical diagnosis, chemical sensors and analysis ${ }^{17,32-37}$. Recently, the MOFs/ZIFs have been used to load enzymes/ proteins into their pores for enhancing the activity, recyclability and solvent adaptability of enzymes/proteins ${ }^{38}$.

Key Laboratory of Functional Small Organic Molecule, Ministry of Education, College of Chemistry and Chemical Engineering, Jiangxi Normal University, 99 Ziyang Road, Nanchang 330022, People's Republic of China. Correspondence and requests for materials should be addressed toY.S. (email: yhsong@jxnu.edu.cn) or L.W. (email: Iwanggroup@aliyun.com) 

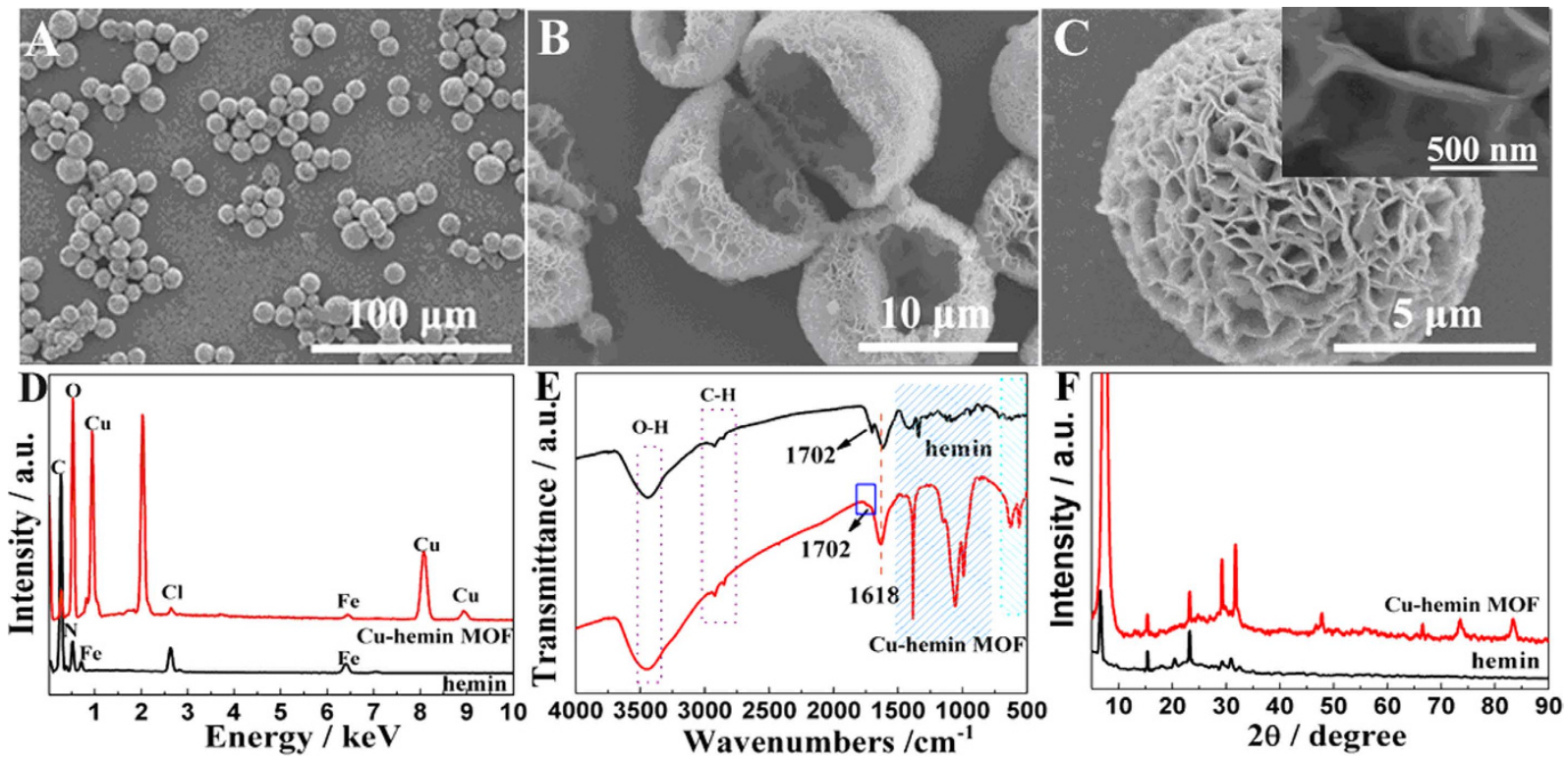

Figure 1. SEM images of $\mathrm{Cu}$-hemin MOFs prepared at the mole ratio of $\mathrm{Cu}^{2+}$ and hemin of 171:1 at (A) Lowand $(\mathbf{B}, \mathbf{C})$ high-magnification. Insert in Fig. 1C showed detailed structure. (D) EDXS, (E) FT-IR spectra and (F) XRD patterns of hemin and $\mathrm{Cu}$-hemin MOFs.

A series of MOFs with differently sized pores were also synthesized via changing the chain length of ligands for loading various biomolecules ${ }^{39-42}$. Some biomolecule@MOFs/ZIFs have been applied for biosensing. For example, Liu Y. et al. synthesized hemin@MIL-101(Al)- $\mathrm{NH}_{2}$ to combine with GOD for colorimetric detection of glucose ${ }^{43}$. Liu Z. et al. has also prepared cytochrome c/ZIF- 8 by one-pot synthesis for glucose and $\mathrm{H}_{2} \mathrm{O}_{2}$ detection by colorimetric method ${ }^{42}$. The previous results clearly indicated that small molecules and electrolyte could still enter into the pores of MOFs/ZIFs freely after the biomolecules was loaded into their pores. However, only a few enzymes are loaded into the MOFs/ZIFs, which results in a narrow linear range. These studies activate us to construct an electrochemical glucose biosensor by using MOFs as immobilizing matrix to load GOD.

Herein, a novel $\mathrm{Cu}$-hemin MOFs with an excellent catalytic activity toward the reduction of $\mathrm{O}_{2}$ was fabricated via $\mathrm{Cu}^{2+}$ coordinating with hemin for electrochemical glucose biosensing. The novel Cu-hemin MOFs showed a 3D ball-flower-like nanostructure and was hollow inside, which could be used to load a large number of GOD molecules. The GOD molecules were incorporated into $\mathrm{Cu}$-hemin MOFs by utilizing the associated pores of MOFs, which effectively avoided the aggregation of enzyme on the surface of electrode. The ball-flower-like nanostructure of $\mathrm{Cu}$-hemin MOFs resulted in the contact of GOD molecules with glucose and electrolyte to keep the catalytic activity of enzyme. The GOD/Cu-hemin MOF nanocomposites could not only catalyze the reduction of $\mathrm{O}_{2}$ via the $\mathrm{Cu}$-hemin MOFs effectively but also catalyze the oxidization of glucose via GOD, avoiding the shortcomings of traditional nanomaterials and other MOFs/ZIFs. The as-prepared glucose sensor based on the GOD/Cu-hemin MOFs exhibited wide linear range, low detection limit, excellent sensitivity and good selectivity.

\section{Results and Discussion}

As shown in Fig. 1A, a large number of uniform 3D Cu-hemin MOFs with ball-flower-like structure appeared. From the low magnification scanning electron microscopy (SEM) image (Fig. 1A), it could be seen that the diameter of the 3D flower-like ball was about $10 \mu \mathrm{m}$. Meanwhile, it could also be seen that the Cu-hemin MOFs showed a big hollow cage and a large number of holes in the flower-like ball, which could not only provide a large specific surface area but also enhance the mass transfer (Fig. 1B). Moreover, the details of the fold structure could be distinctly revealed by the high magnification SEM image (Fig. 1C) and the average thickness of these flakes was about $50 \mathrm{~nm}$ (insert of Fig. 1C). Due to different linkages between metal ions and organic ligands, MOFs could present various morphologies including rod octahedron ${ }^{42,44}$, nanosphere ${ }^{45}$, hexahedron ${ }^{45,46}$, spindle-shape $\mathrm{e}^{47}$, and so on. However, such ball-flower-like structure has not yet been reported in previous works. The special structure of hemin might result in the formation of such ball-flower-like structure via a linkage between $\mathrm{Cu}^{2+}$ and carboxy groups of hemin. As a fixed-structure molecule, hemin owned three feature elements $(\mathrm{Fe}, \mathrm{N}$ and $\mathrm{Cl})$ which could be used to prove its existence ${ }^{36,48,49}$. As could be seen from energy dispersive X-ray spectroscopy in Fig. 1D, the apparent peaks of $\mathrm{Fe}, \mathrm{N}, \mathrm{Cl}$ and $\mathrm{Cu}$ were observed in the solid products, which declared the formation of $\mathrm{Cu}$-hemin MOFs. In order to verify this conclusion, Fourier transform infrared spectroscopy (FT-IR) and the X-ray powder diffraction (XRD) test were carried out. In the FT-IR spectra (Fig. 1E), both hemin and $\mathrm{Cu}$-hemin MOFs exhibited obvious peaks for $\mathrm{O}-\mathrm{H}$ at $3442 \mathrm{~cm}^{-1}$. The band at $1618 \mathrm{~cm}^{-1}$ originated from $\mathrm{C}=\mathrm{C}$ and $\mathrm{C}=\mathrm{N}$ of the protoporphyrin (IX) ring system ${ }^{25}$. The weak peak at $1701 \mathrm{~cm}^{-1}$ was ascribed to $\mathrm{C}=\mathrm{O}$ stretching vibration, which was smaller in the curve of $\mathrm{Cu}$-hemin $\mathrm{MOF}$ s due to the coordination between $-\mathrm{COOH}$ groups and $\mathrm{Cu}^{2+}$. The strong peak at $1384 \mathrm{~cm}^{-1}$ belonged to $-\mathrm{CH}_{3}$ of hemin was stronger in the curve of $\mathrm{Cu}$-hemin MOFs due to the ordered assembly of hemin in the Cu-hemin $\mathrm{MOFs}^{50}$. Hence, FT-IR spectra also proved the 

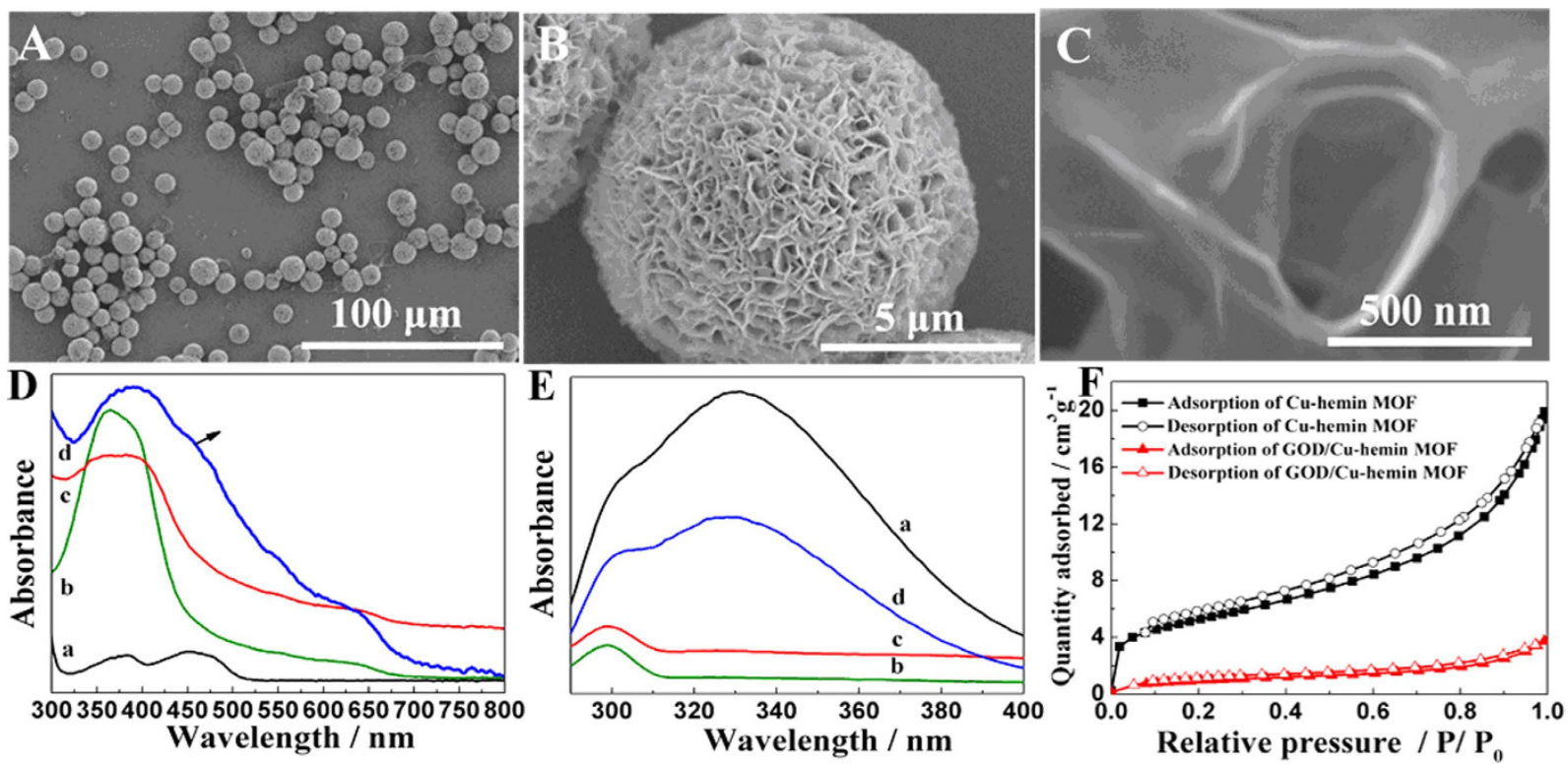

Figure 2. SEM images of GOD/Cu-hemin MOFs nanocomposites at (A) Low- and (B,C) high-magnification. (D) UV-vis spectra and (E) Fluorescence spectra of GOD (a), hemin (b), Cu-hemin MOFs (c) and GOD/Cuhemin MOFs nanocomposites (d). (F) $\mathrm{N}_{2}$ adsorption/desorption isotherms measured at $77 \mathrm{~K}$ for the $\mathrm{Cu}$-hemin MOFs (black curve) and GOD/Cu-hemin MOFs nanocomposites (red curve).

formation of $\mathrm{Cu}$-hemin MOFs. The XRD spectra of hemin and $\mathrm{Cu}$-hemin MOFs were exhibited in Fig. 1F $\mathrm{F}^{51,52}$. Some diffraction peaks appeared in the pattern of Cu-hemin MOFs, suggesting that the 3D-flower-like ball nanostructures was crystalline materials and the crystal structure of $\mathrm{Cu}$-hemin MOFs was similar to that of previous hemin-MOFs ${ }^{37}$. The XPS of Cu-hemin MOFs exhibited in Figure S1 (Supporting Information) has also proved the successful synthesis of $\mathrm{Cu}$-hemin MOFs. The detailed discussion on the crystal structure of $\mathrm{Cu}$-hemin MOFs will be reserved in the future study. All the results confirmed that the 3D-flower-like ball nanostructures was the $\mathrm{Cu}$-hemin MOFs which had an ordered structure and high crystalline.

The SEM images of GOD/Cu-hemin MOFs nanocomposites showed a similar diameter and morphology to that of $\mathrm{Cu}$-hemin MOFs (Fig. 2A). Magnified SEM images further confirmed that the Cu-hemin MOFs maintained its framework integrity after loading GOD molecules in the pores (Fig. 2B,C), which was also confirmed by the XRD studies (Figure S2A, Supporting Information). The UV-vis absorbance spectrum of hemin (curve b, Fig. 2D) revealed a strong absorption peak at $367 \mathrm{~nm}$ of Soret band and a weak absorption peak at about $652 \mathrm{~nm}$ of Q-band ${ }^{25,52}$. Two absorption peaks also appeared at the spectrum of Cu-hemin MOFs (curve c) and GOD/ $\mathrm{Cu}$-hemin MOFs nanocomposites (curve d), indicating the structure of hemin was maintained. A doublet peaks at $375 \mathrm{~nm}$ and $455 \mathrm{~nm}$ appeared in the spectrum of GOD (curve a) ) $^{7,53}$. For GOD/Cu-hemin MOFs nanocomposites, a weak peak at $457 \mathrm{~nm}$ was observed and the peak at $375 \mathrm{~nm}$ might be covered by the strong peak at $367 \mathrm{~nm}$, which indicated that GOD was successfully loaded into $\mathrm{Cu}$-hemin MOFs. No obvious shift of peaks in the GOD/ $\mathrm{Cu}$-hemin MOFs nanocomposites was observed (curve d), which might indicate the structure of GOD was well kept. Since the carboxyl groups of hemin were coordinated with $\mathrm{Cu}^{2+}$ to form $\mathrm{Cu}$-hemin MOFs, the amine of GOD could not link with carboxyl groups of hemin and accordingly GOD molecules might be loaded in the pores of $\mathrm{Cu}$-hemin MOFs. Figure 2E displayed a steady-state fluorescence spectra of these materials, and it revealed that only GOD (curve a) and GOD/Cu-hemin MOFs nanocomposites (curve d) showed strong emission peak at $339 \mathrm{~nm}$. The decrease of density in fluorescence spectrum of GOD/Cu-hemin MOFs nanocomposites might indicate that GOD molecules were loaded in the pores of $\mathrm{Cu}$-hemin MOFs. $\mathrm{N}_{2}$ adsorption/desorption isotherms (Fig. 2F) test under $77 \mathrm{~K}$ were carried out and showed that the Brunauer-Emmett-Teller (BET) surface area of $\mathrm{Cu}$-hemin MOFs decreased from 17.00 to $3.08 \mathrm{~m}^{2} \mathrm{~g}^{-1}$ after the loading of GOD. The result further confirmed that GOD molecules were loaded in the pores of $\mathrm{Cu}$-hemin MOFs. The FT-IR spectra and thermogravimetric data (TGA) curves (Figure S2B,C, Supporting information) also confirmed the conclusion.

Electrochemical properties of $\mathrm{Cu}$-hemin MOFs/glassy carbon electrode (GCE) and GOD/Cu-hemin MOFs nanocomposites/GCE at different scan rates were investigated by cyclic voltammograms (CVs) which both showed a pair of distinct redox peak of hemin (Fig. 3A,B $)^{15,36,48,54}$. The peak currents $\left(I_{\mathrm{p}}\right)$ displayed good linear correlations with scan rates $(v)$ from 20 to $150 \mathrm{mV} \mathrm{s}^{-1}$, suggesting quasi-reversible surface-controlled processes (Fig. 3C,D). The results clearly indicated that electrochemical properties of hemin were not changed in both $\mathrm{Cu}$-hemin MOFs and GOD/Cu-hemin MOFs nanocomposites. It could be ascribed to the porous structure of $\mathrm{Cu}$-hemin MOFs and GOD/Cu-hemin MOFs nanocomposites which enhanced the mass transfer effectively. After GOD was loaded in $\mathrm{Cu}$-hemin MOFs, the peak current decreased slightly, indicating GOD molecules was successfully loaded in the pores of $\mathrm{Cu}$-hemin MOFs. In the potential range, the typical redox peaks of GOD molecules were not observed. It is well known that the redox center of GOD is obstructed by the protein shell and the electron transport rate between the active site of GOD and electrode surface is slow. The direct electron 

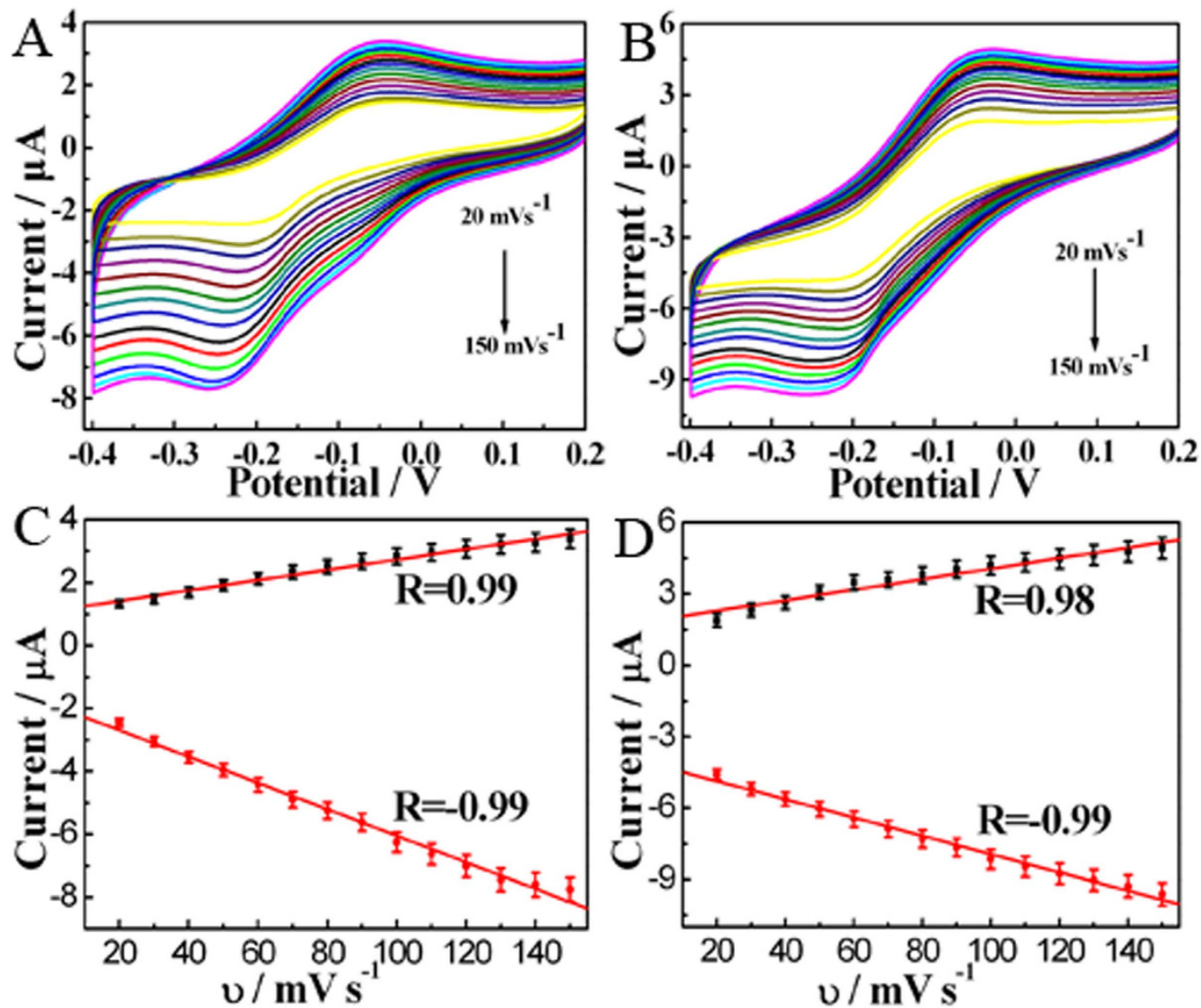

Figure 3. CVs of (A) Cu-hemin MOFs/GCE and (B) GOD/Cu-hemin MOFs/GCE in $0.1 \mathrm{M} \mathrm{N}_{2}$-saturated PBS $(\mathrm{pH}=7.0)$ at different scan rates by step of $10 \mathrm{mV} \mathrm{s}^{-1}$. Plot of peak current versus the scan rates for $(\mathbf{C}) \mathrm{Cu}-$ hemin MOFs/GCE and (D) GOD/Cu-hemin MOFs nanocomposites/GCE.

transfer could be achieved by immobilizing GOD molecules on nanomaterials where the secondary structure of GOD molecules was changed to reveal the eletroactive center but their bioactivity was lost ${ }^{19}$. Thus, the missing of redox peaks for GOD molecules might indicate their bioactivity was well kept in the GOD/Cu-hemin MOFs nanocomposites.

Electrochemical impedance spectroscopy (EIS) was used to explore the electron transfer of various electrodes and the results were shown in Figure S3A (Supporting Information). The Randles circuit (Inset of Figure S3A, Supporting Information) was used for matching with the impedance records. The resistance of charge transfer $\left(R_{c t}\right)$ of the bare GCE ( $147 \Omega$, curve a) as indicated by the Nyquist circle on the curve was very small. The $R_{c t}$ of $\mathrm{Cu}$-hemin MOFs/GCE ( $3079 \Omega$, curve b) increased greatly due to its poor conductivity. After the loading of GOD, the $R_{c t}$ was further increased to $7094 \Omega$ (curve c) which might be attributed to the blocking effects of negatively charged biomacromolecule of GOD on $\mathrm{Fe}(\mathrm{CN})_{6}{ }^{3-/ 4-}$. The result also confirmed that GOD molecules were successfully loaded in the pores of $\mathrm{Cu}$-hemin MOFs.

Since most of enzymatic glucose biosensors are used to detect glucose level via monitoring the consumption of $\mathrm{O}_{2}$ in enzymatic reaction, it is very important to explore the electrocatalytic activity of GOD/Cu-hemin MOFs nanocomposites and $\mathrm{Cu}$-hemin MOFs toward ORR. Figure 4A,B showed CVs of Cu-hemin MOFs/GCE and GOD/Cu-hemin MOFs nanocomposites/GCE in $0.1 \mathrm{M} \mathrm{N}_{2} / \mathrm{O}_{2}$-saturated PBS at a scan rate of $50 \mathrm{mV} \mathrm{s}^{-1}$ for oxygen reduction reaction (OOR), respectively. As shown in Fig. 4A, the anodic peak was diminishing with the increased $\mathrm{O}_{2}$ in electrolytes and the reductive peak became obvious with a slight positive shift, showing a typical ORR. After GOD molecules were loaded on the $\mathrm{Cu}$-hemin MOFs, the reductive peak current became larger and the peak potential positively shifted as compared with that of the $\mathrm{Cu}$-hemin MOFs (Fig. 4B), indicating GOD might be also involved in the catalytic process. The reductive peak current of GOD/Cu-hemin MOFs nanocomposites/GCE was also larger than that of hemin/GCE where the same amount of hemin was modified on the electrode surface (Figure S3B, Supporting Information), suggesting the electrocatalytic performance toward $\mathrm{O}_{2}$ was greatly enhanced when hemin was used to construct the $\mathrm{Cu}$-hemin MOFs. The large peak current could be used as significantly amplified signal for glucose detection. The effect of GOD amount on the electrocatalytic performance toward $\mathrm{O}_{2}$ was studied by the cathodic peak on CVs (Figure S4A, Supporting Information), which indicated the maximum cathodic peak was at $1.5 \mathrm{nmol}$ GOD. In this work, the influence of $\mathrm{pH}$ was also explored from 6.0 to 8.0 (Figure S4B, Supporting Information), which displayed a optimum 

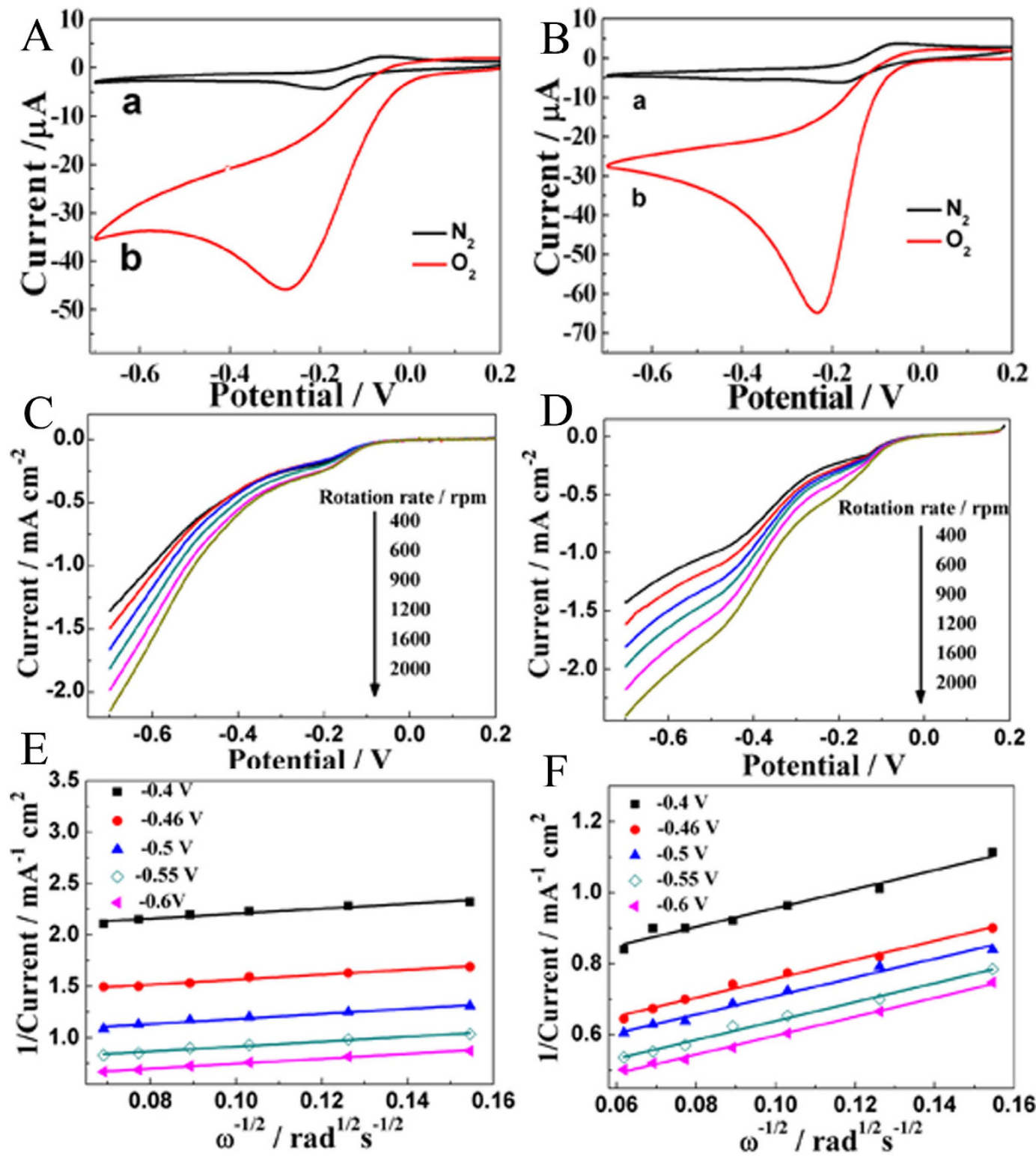

Figure 4. CVs of (A) Cu-hemin MOFs/GCE and (B) GOD/Cu-hemin MOFs/GCE in $0.1 \mathrm{M} \mathrm{N}_{2}$-saturated (black curve) and $\mathrm{O}_{2}$-saturated (red curve) PBS ( $\mathrm{pH}=7.0$ ). Scan rate: $50 \mathrm{mV} \mathrm{s}^{-1}$. RDE LSV of $(\mathbf{C}) \mathrm{Cu}$-hemin MOFs/ GCE and (D) GOD/Cu-hemin MOFs/GCE in $0.1 \mathrm{M} \mathrm{O}_{2}$-saturated $\mathrm{PBS}(\mathrm{pH}=7.0)$ with various rotation rates. Koutecky-Levich plots at different electrode potentials for (E) Cu-hemin MOFs/GCE and (F) GOD/Cu-hemin MOFs/GCE.

electrocatalytic current at $\mathrm{pH}$ 7.0. It could be ascribed to the fact that the optimal $\mathrm{pH}$ of the enzymatic reaction was $\mathrm{pH} 7.0$ (that is, the bioactivity of GOD was largest at $\mathrm{pH} 7.0$ ).

To obtain the electron transfer number of ORR by $\mathrm{Cu}$-hemin MOFs and GOD/Cu-hemin MOFs nanocomposites, reaction kinetics were investigated by rotating-disk electrode (RDE) linear sweep voltammograms (LSV). The current density was enhanced by increasing of rotation rate from 400 to $2000 \mathrm{rpm}$ (Fig. 4C,D). The corresponding Koutecky- Levich plots $\left(J^{-1}\right.$ vs $\left.\omega^{-1 / 2}\right)$ under different electrode potentials showed a good linearity (Fig. 4E,F). Linearity and parallelism of the plots were considered as typical of first-order reaction kinetics with respect to the dissolved $\mathrm{O}_{2}$. The kinetic parameters could be calculated based on the Koutecky-Levich equations: ${ }^{25}$

$$
\begin{gathered}
\frac{1}{J}=\frac{1}{J_{K}}+\frac{1}{J_{L}}=\frac{1}{J_{K}}+\frac{1}{B \omega^{\frac{1}{2}}} \\
B=0.62 n F C_{0}\left(D_{0}\right)^{\frac{2}{3}} \nu^{-\frac{1}{6}}
\end{gathered}
$$



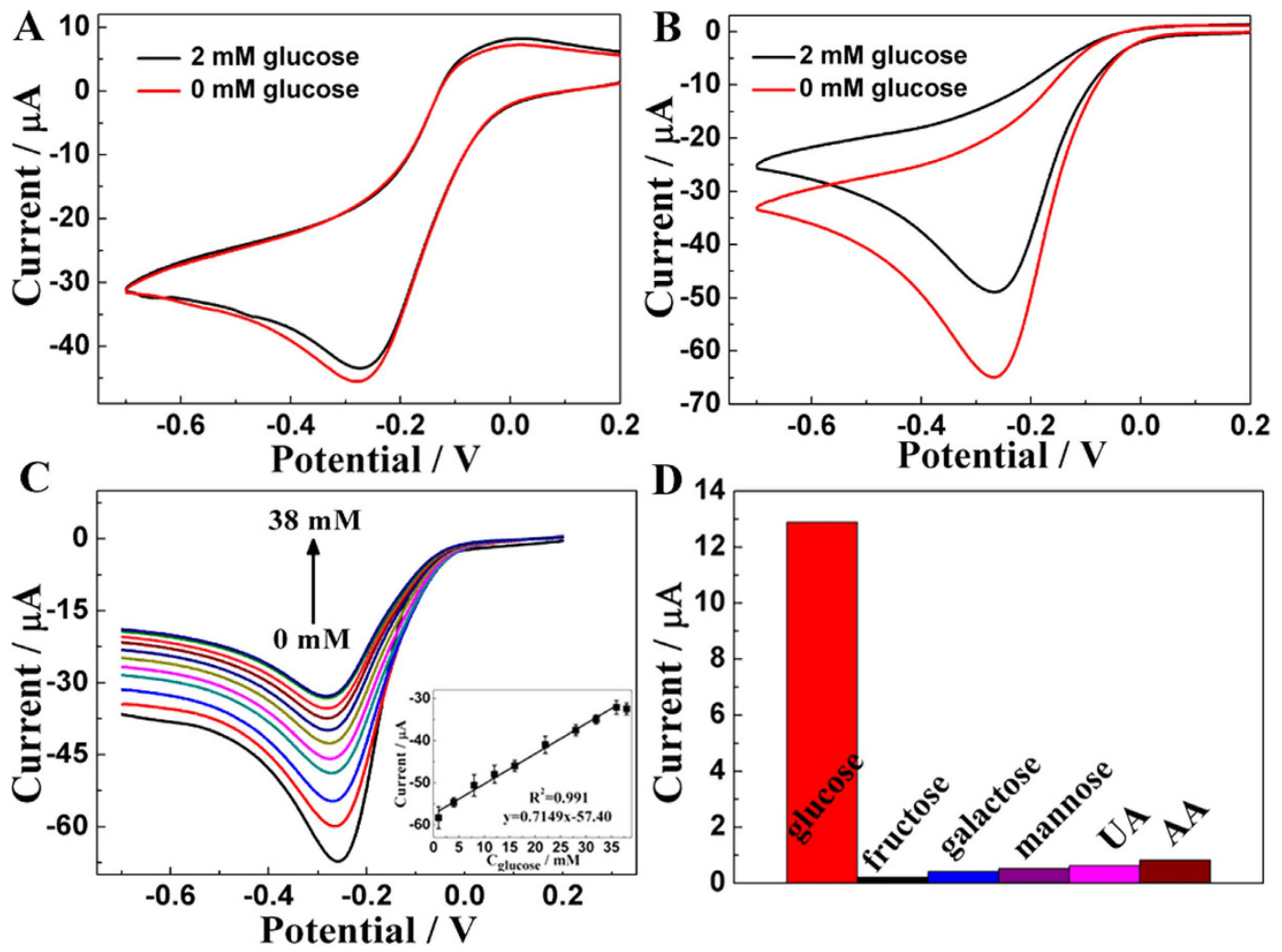

Figure 5. (A) Cu-hemin MOFs/GCE and (B) GOD/Cu-hemin MOFs/GCE in $0.1 \mathrm{M} \mathrm{O}_{2}$-saturated PBS $(\mathrm{pH}=7.0)$ in the absence (red curve) and presence (black curve) of $2 \mathrm{mM}$ glucose. (C) LSV of GOD/Cu-hemin $\mathrm{MOF} / \mathrm{GCE}$ in $0.1 \mathrm{M} \mathrm{O}_{2}$-saturated PBS $(\mathrm{pH}=7.0)$ in the presence of glucose with various concentrations. Insert: Plot of peak current versus the concentration of glucose. (D) Comparison of the change of CVs response of GOD/Cu-hemin MOFs/GCE in $0.1 \mathrm{M} \mathrm{O}_{2}$-saturated PBS $(\mathrm{pH}=7.0)$ containing $0.2 \mathrm{mM}$ glucose and $2 \mathrm{mM}$ various interferences. Scan rate: $50 \mathrm{mV} \mathrm{s}^{-1}$.

$$
J_{K}=n F k C_{0}
$$

in which $J$ is the measured current density, $J_{K}$ and $J_{L}$ are the kinetic and diffusion-limiting current densities, $\omega$ is the angular velocity of the disk ( $\omega=2 \pi \mathrm{N}, \mathrm{N}$ is the linear rotation speed), $n$ is the overall number of electrons transferred in oxygen reduction, $F$ is the Faraday constant $\left(F=96485 \mathrm{C} \mathrm{mol}^{-1}\right), C_{0}$ is the bulk concentration of $\mathrm{O}_{2}, \nu$ is the kinematic viscosity of the electrolyte, $D_{0}$ is the coefficient of diffusion for $\mathrm{O}_{2}$, and the $k$ is the electron transfer rate constant. $n$ and $J_{K}$ could be obtained from the slope and intercept of the Koutecky- Levich plots in Fig. $4 \mathrm{E}, \mathrm{F}$ (parameters $C_{0}=1.2 \times 10^{-3} \mathrm{~mol} \mathrm{~L}^{-1}, D_{0}=1.9 \times 10^{-5} \mathrm{~cm} \mathrm{~s}^{-1}$, and $v=0.01 \mathrm{~cm}^{2} \mathrm{~s}^{-1}$ in $0.1 \mathrm{M} \mathrm{PBS}$ ), respectively. Then $n$ was estimated to be 3.78 and 3.41 for $\mathrm{Cu}$-hemin MOFs and GOD/Cu-hemin MOFs nanocomposites, respectively. The result indicated that a direct 4-electron reduction pathway was obtained for ORR.

In order to further discuss whether GOD/Cu-hemin MOFs nanocomposites/GCE could catalyze glucose, a study was carried out in the presence and absence of $2 \mathrm{mM}$ glucose in $0.1 \mathrm{M} \mathrm{O}_{2}$-saturated PBS (Fig. 5A,B). It displayed an obvious decrease of cathodic current at the GOD/Cu-hemin MOFs nanocomposites/GCE (Fig. 5B) while only a slight decrease of cathodic current occurred at the $\mathrm{Cu}$-hemin MOFs/GCE (Fig. 5A), exhibiting a typical electrocatalysis of glucose by GOD. The good catalytic performance might result from both a large number of GOD loaded in the $\mathrm{Cu}$-hemin MOFs effectively and the good bioactivity of GOD remained in Cu-hemin MOFs. In Figure S5A,B (Supporting Information), CVs was carried out to further explore the bioactivity of GOD, where the current change was similar to free GOD and GOD/Cu-hemin MOF nanocomposite after glucose was added into the electrolyte solution. Meanwhile, a wide linear range of GOD/Cu-hemin MOFs/GCE from $9.10 \mu \mathrm{M}$ to $36.0 \mathrm{mM}(R=0.99, \mathrm{~S} / \mathrm{N}=3)$ was achieved (Fig. $5 \mathrm{C})$. And the detection limit was estimated to be $2.73 \mu \mathrm{M}$ with a higher sensitivity of about $22.77 \mu \mathrm{A} \mathrm{mM}^{-1} \mathrm{~cm}^{-2}$. All these results exhibited superiority to other GOD-based glucose sensor as compared in Table S1 (Supporting Information).

Figure 5D showed chemicals including fructose, galactose, mannose, uric acid (UA) and ascorbic acid (AA) in ten times concentration of glucose did not interfere with glucose detection. The result indicated that the GOD/ $\mathrm{Cu}$-hemin MOFs nanocomposite/GCE had a perfect selectivity for glucose detection. As shown in Table S2 (Supporting Information), the detection of glucose in human serum sample was studied by adding glucose into human serum solution which was diluted by $0.1 \mathrm{M}$ PBS. The results of recovery indicated that the GOD/Cu-hemin MOFs nanocomposite/GCE was reliable and sensitive enough for real sample detection. The concentration of 
glucose in human serum sample of diabetes mellitus patients has also been evaluated to be about $8.32 \mathrm{mM}$, close to the value obtained from the clinical results with a relative deviation of $0.43 \%$.

The long-term stability of GOD/Cu-hemin MOFs nanocomposite/GCE was explored, and the biosensor retained $99.6 \%$ of original current after the biosensor was stored at $4{ }^{\circ} \mathrm{C}$ for $48 \mathrm{~h}$ and $87.5 \%$ of original current for 30 days (Figure S6, Supporting Information). The reproducibility of the biosensors was also evaluated from the response toward $2 \mathrm{mM}$ glucose at with five electrodes and the relative standard deviation (RSD) was about $4.27 \%$. The results indicated that the biosensor showed a good long-term stability and reproducibility for glucose detection.

In summary, a novel ball-flower-like Cu-hemin MOFs with excellent catalytic activity toward the reduction of $\mathrm{O}_{2}$ was fabricated via the coordination of $\mathrm{Cu}^{2+}$ with hemin for the first time. The porous Cu-hemin MOFs could be used as supporting materials to load GOD effectively for fabricating glucose biosensor. The porous hollow structure of GOD/Cu-hemin MOFs nanocomposites enhanced the mass transfer and improved the efficiency of active GOD molecules, and the bioactivity of GOD could also be kept in the GOD/Cu-hemin MOFs nanocomposites. The GOD/Cu-hemin MOFs nanocomposites could not only catalyze the reduction of $\mathrm{O}_{2}$ via the $\mathrm{Cu}$-hemin MOFs but also catalyze the oxidization of glucose via GOD, which successfully avoided the drawback of nanomaterials as supporting to load GOD. As a consequence, the as-prepared glucose sensor based on the GOD/Cu-hemin MOFs nanocomposites exhibited wide linear range, low detection limit, excellent sensitivity and good selectivity. Overall, the proposed method to prepare $\mathrm{Cu}$-hemin MOFs nanocomposites is simple, efficient and easy to mass production. It might open up a new way for glucose sensors and shed new light on MOFs-based biosensors.

\section{Methods \\ Materials. Hemin and copper nitrate trihydrate $\left(\mathrm{Cu}\left(\mathrm{NO}_{3}\right)_{2} \cdot 3 \mathrm{H}_{2} \mathrm{O}\right)$ were purchased from Aladdin Reagent Co., Ltd (Shanghai, China). Glucose oxidase (GOD, EC 1.1.3.4, $140 \mathrm{U} \mathrm{mg}^{-1}$ ) and human serum were purchased from Sigma-Aldrich. The other chemicals were obtained from Beijing Chemical Reagent Factory (Beijing, China) and were of analytical grade without further purification. Phosphate buffer solution (PBS, $0.1 \mathrm{M}$ ) was prepared by mixing $0.2 \mathrm{M} \mathrm{Na}_{2} \mathrm{HPO}_{4}$ and $0.2 \mathrm{M} \mathrm{NaH}_{2} \mathrm{PO}_{4}$. Hemin solution $(0.50 \mathrm{mM})$ was obtained by ultrasonic dissolving hemin in $0.1 \mathrm{M} \mathrm{NaOH}$ then the $\mathrm{pH}$ was adjusted to 7.0. GOD solutions $(62.5 \mu \mathrm{M})$ were obtained by dissolving GOD in $0.1 \mathrm{M} \mathrm{PBS}$ ( $\mathrm{pH} 7.0$ ). The solution of hemin and GOD were stored in refrigerator before use. Ultra-pure water was purified by a Millipore-Q System $\left(\rho \geq 18.2 \mathrm{M} \Omega \mathrm{cm}^{-1}\right)$ and used in the whole experiments.}

Instrumentation. SEM and EDXS characterizations were operated on a HITACHI S-3400N scanning electron microscope with a Phoenix energy X-ray analyzer. XRD data were collected on a D/Max 2500 V/PC $\mathrm{X}$-ray powder diffractometer using $\mathrm{Cu} \mathrm{K} \alpha$ radiation $(\lambda=0.154056 \mathrm{~nm}, 40 \mathrm{kV}, 200 \mathrm{~mA})$. FT-IR spectroscopy was obtained by a Perkin-Elmer Spectrome 100 spectrometer (Perkin-Elmer Company, USA) with KBr power. $\mathrm{N}_{2}$ adsorption/desorption isotherms were measured at $77 \mathrm{~K}$ in a liquid nitrogen atmosphere using a Tristar 3000 volumetric adsorption analyzer (Micromeritics Instrument Corporation, USA) after the samples were pretreated at $200^{\circ} \mathrm{C}$ for $12 \mathrm{~h}$ under vacuum. TGA were conducted on SDT 2960 with a heating rate of $10^{\circ} \mathrm{C} \mathrm{min}{ }^{-1}$ under $\mathrm{N}_{2}$. UV-vis absorption spectra were recorded on a Hitachi U-3900H UV-vis Spectrophotometer. Fluorescence spectroscopy was determined on a Fluorescence Spectrophotometer (Hitachi) F-7000.

All electrochemical measurements were performed on a CHI 660C electrochemical workstation (Shanghai, China) at ambient temperature. A common three electrode system was employed including a bare or modified GCE as the working electrode, a platinum wire as the auxiliary electrode and a saturated calomel electrode (SCE) as the reference electrode. CVs and LSV were performed in a quiescent $0.1 \mathrm{M} \mathrm{PBS} \mathrm{(pH} \mathrm{7.0).} \mathrm{EIS} \mathrm{results}$ were operated in $5 \mathrm{mM} \mathrm{Fe}(\mathrm{CN})_{6}{ }^{3-14-}$ solution including $0.1 \mathrm{M} \mathrm{KCl}$ as the supporting electrolyte. Rotating disk electrode (RDE) measurements were performed in $\mathrm{O}_{2}$-saturated $0.1 \mathrm{M}$ PBS ( $\mathrm{pH}$ 7.0) solution with rotation rates from 400 to $2000 \mathrm{rpm}$ at a scan rate of $10 \mathrm{mV} \mathrm{s}^{-1}$ on a GC disk electrode (Pine Instruments, $5.0 \mathrm{~mm}$ in diameter, $\mathrm{A}=0.2 \mathrm{~cm}^{2}$ ) as working electrode, a Pt plate as the counter electrode and a $\mathrm{Ag} / \mathrm{AgCl}$ (saturated $\mathrm{KCl}$ ) as the reference electrode.

Preparation of of GOD/Cu-hemin MOFs Nanocomposites. $\quad 0.04 \mathrm{M} \mathrm{Cu}\left(\mathrm{NO}_{3}\right)_{2} \cdot 3 \mathrm{H}_{2} \mathrm{O}$ solution and $0.50 \mathrm{mM}$ hemin solution were mixed together with a volume ratio of 2:1 at room temperature and reacted for about $2 \mathrm{~h}$. The produced ash green powder was acquired by centrifugation, washed with water and dried at $50^{\circ} \mathrm{C}$ overnight. Then $24 \mu \mathrm{L} 62.5 \mu \mathrm{M}$ GOD was added into $150 \mu \mathrm{L}$ suspension of $\mathrm{Cu}$-hemin MOFs for $24 \mathrm{~h}$ at $4{ }^{\circ} \mathrm{C}$. The resulted GOD/Cu-hemin MOFs powder was acquired by centrifugation, washed with $0.1 \mathrm{M} \mathrm{PBS}(\mathrm{pH} 7.0)$ and dried at room temperature for overnight. The conditions to prepare $\mathrm{Cu}$-hemin MOFs and GOD/Cu-hemin MOFs was optimized and the results were shown in Figure S7 (Supporting Information). As shown in Figure S7, as the ratio of $\mathrm{Cu}^{2+}$ and hemin was increased from 10:1 to 171:1 (Figure S7A-C, Supporting Information), the nanostructure became more and more uniform and finally some uniform ball-flower-like nanostructures appeared. After the mole ratio of $\mathrm{Cu}^{2+}$ and hemin was increased to 513:1 (Figure S7D, Supporting information), some nanostructures were damaged. Although some small Cu-hemin MOFs could be observed at 10:1, the Cu-hemin MOFs was not uniform and some deformed $\mathrm{Cu}$-hemin MOFs were also observed, which was bad for the electrochemical performance. Therefore, the $\mathrm{Cu}$-hemin MOFs prepared at the mole ratio of $\mathrm{Cu}^{2+}$ and hemin of 171:1 was used to construct the electrochemical biosensors.

Preparation of the Modified Electrode. The bare GCE ( $3 \mathrm{~mm}$ in diameter) was polished with 1.0 and $0.3 \mu \mathrm{m}$ alumina powder, ultrasonic washed in pure water for $5 \mathrm{~min}$, then dried under a high-purity $\mathrm{N}_{2}$ stream. The modified GCE were prepared by dropping $9 \mu \mathrm{L} \mathrm{Cu}$-hemin MOFs or GOD/Cu-hemin MOFs aqueous suspension on the surface of the polished GCE and then dried at $4{ }^{\circ} \mathrm{C}$. Next, $1.0 \mu \mathrm{L}$ of $0.05 \%$ nafion solution were 
dropped onto the modified GCE surface and subsequently dried at $4{ }^{\circ} \mathrm{C}$ for $4 \mathrm{~h}$. Finally, the modified electrode was immersed into $0.1 \mathrm{M}$ PBS (pH 7.0) to remove those weakly bound molecules and obtain the nanocomposites modified target electrode.

\section{References}

1. Li, M. et al. Facile synthesis of ultrafine $\mathrm{Co}_{3} \mathrm{O}_{4}$ nanocrystals embedded carbon matrices with specific skeletal structures as efficient non-enzymatic glucose sensors. Anal. Chim. Acta 861, 25-35 (2015).

2. Bai, L. et al. Carbon nanotubes-ionic liquid nanocomposites sensing platform for NA DH oxidation and oxygen, glucose detection in blood. Talanta 91, 110-115 (2012).

3. Wang, X. Z. et al. Highly sensitive and selective electrochemical identification of d-glucose based on specific concanavalin A combined with gold nanoparticles signal amplification. Sensor. Actuator. B 185, 105-109 (2013).

4. Lin, Y. Q. et al. Continuous and simultaneous electrochemical measurements of glucose, lactate, and ascorbate in rat brain following brain ischemia. Anal. Chem. 86, 3895-3901 (2014).

5. Welch, M. E. et al. A glucose sensor via stable immobilization of the GOx enzyme on an organic transistor using a polymer brush. J. Polym. Sci. Part A: Polym. Chem. 53, 372-377 (2015).

6. Ariga, K. et al. Enzyme nanoarchitectonics: organization and device application. Chem. Soc. Rev. 42, 6322-6345 (2013).

7. Song, Y. H., Liu, H. Y., Wang, Y. \& Wang, L. A novel bi-protein bio-interphase of cytochrome c and glucose oxidase: Electron transfer and electrocatalysis. Electrochim. Acta 93, 17-24 (2013).

8. Lu, W. B. et al. Ni foam: a novel three-dimensional porous sensing platform for sensitive and selective nonenzymatic glucose detection. Analyst 138, 417-420 (2013).

9. Chen, H. C. et al. Direct electron transfer of glucose oxidase and dual hydrogen peroxide and glucose detection based on waterdispersible carbon nanotubes derivative. Anal. Chim. Acta. 867, 83-91 (2015).

10. Hu, C. Y. et al. Enzyme-labeled Pt@BSA nanocomposite as a facile electrochemical biosensing interface for sensitive glucose determination. ACS Appl. Mater. Inter. 6, 4170-4178 (2014).

11. Song, Y. H. et al. pH-switchable electrochemical sensing platform based on chitosan-reduced graphene oxide/concanavalin a layer for assay of glucose and urea. Anal. Chem. 86, 1980-1987 (2014).

12. Qin, X.Y. et al. Green, low-cost synthesis of photoluminescent carbon dots by hydrothermal treatment of willow bark and their application as an effective photocatalyst for fabricating Au nanoparticles-reduced graphene oxide nanocomposites for glucose detection. Catal. Sci. Technol. 3, 1027-1035 (2013).

13. Xiang, D. et al. Amperometric hydrogen peroxide and glucose biosensor based on $\mathrm{NiFe}_{2} /$ ordered mesoporous carbon nanocomposites. Analyst 140, 644-653 (2015).

14. Guo, X. S. et al. Glucose biosensor based on a platinum electrode modified with rhodium nanoparticles and with glucose oxidase immobilized on gold nanoparticles. Microchim. Acta 181, 519-525 (2014).

15. Amanullah, S., Das, P. K., Samanta, S. \& Dey, A. Tuning the thermodynamic onset potential of electrocatalytic $\mathrm{O}_{2}$ reduction reaction by synthetic iron-porphyrin complexes. Chem. Commun. 51, 10010-10013 (2015).

16. Yang, C., Xu, C. X. \& Wang, X. M. ZnO/Cu nanocomposite: a platform for direct electrochemistry of enzymes and biosensing applications. Langmuir 28, 4580-4585 (2012).

17. Zhu, Q. L., Li, J. \& Xu, Q. Immobilizing metal nanoparticles to metal-organic frameworks with size and location control for optimizing catalytic performance. J. Am. Chem. Soc. 135, 10210-10213 (2013).

18. Qin, X. Y. et al. One-pot synthesis of Au nanoparticles/reduced graphene oxide nanocomposites and their application for electrochemical $\mathrm{H}_{2} \mathrm{O}_{2}$, glucose, and hydrazine sensing. Gold Bulletin. 47, 3-8 (2014).

19. Song, Y. H. et al. Conformation, bioactivity and electrochemical performance of glucose oxidase immobilized on surface of gold nanoparticle. Electrochim. Acta 158, 56-63 (2015).

20. Dong, S. Y. et al. Design synthesis of polypyrrole- $-\mathrm{Co}_{3} \mathrm{O}_{4}$ hybrid material for the direct electrochemistry of hemoglobin and glucose oxidase. Bioelectrochemistry 98, 87-93 (2014).

21. Wooten, M., Karra, S., Zhang, M. G. \& Gorski, W. On the direct electron transfer, sensing, and enzyme activity in the glucose oxidase/carbon nanotubes system. Anal. Chem. 86, 752-757 (2014).

22. Wen, D., Liu, W., Herrmann, A. K. \& Eychmuller, A. A membraneless glucose/O $\mathrm{O}_{2}$ biofuel cell based on Pd aerogels. Chem. Eur. J. 20, 4380-4385 (2014).

23. Zhang, Y. F. et al. Electrocatalytically active cobalt-based metal-organic framework with incorporated macroporous carbon composite for electrochemical applications. J. Mater. Chem. A 3, 732-738 (2015).

24. Guo, Y. J., Li, J. \& Dong, S. J. Hemin functionalized graphene nanosheets-based dual biosensor platforms for hydrogen peroxide and glucose. Sensor. Actuator. B 160, 295-300 (2011).

25. Jahan, M., Bao, Q. L. \& Loh, K. P. Electrocatalytically active graphene-porphyrin MOF composite for oxygen reduction reaction. J. Am. Chem. Soc. 134, 6707-6713 (2012).

26. Li, L.L. et al. Ultrasensitive electrospun nickel-doped carbon nanofibers electrode for sensing paracetamol and glucose. Electrochim. Acta 152, 31-37 (2015).

27. Nayak, P., Santhosh, P. N. \& Ramaprabhu, S. Synthesis of Au-MWCNT-graphene hybrid composite for the rapid detection of $\mathrm{H}_{2} \mathrm{O}_{2}$ and glucose. RSC Adv. 4, 41670-41677 (2014).

28. Kong, C. C. et al. Templating synthesis of hollow $\mathrm{CuO}$ polyhedron and its application for nonenzymatic glucose detection. J. Mater. Chem. A 2, 7306-7312 (2014).

29. Nikolaev, K. et al. A novel bioelectrochemical interface based on in situ synthesis of gold nanostructures on electrode surfaces and surface activation by Meerwein's salt. A bioelectrochemical sensor for glucose determination. Bioelectrochemistry 105, 34-43 (2015).

30. Lu, J. et al. Cobalt[II] schiff base/large mesoporous carbon composite film modified electrode as electrochemical biosensor for hydrogen peroxide and glucose. Electroanalysis 25, 2531-2538 (2013).

31. Tremey, E. et al. Switching an $\mathrm{O}_{2}$ sensitive glucose oxidase bioelectrode into an almost insensitive one by cofactor redesign. Chem. Commun. 50, 5912-5914 (2014).

32. Ma, W. J. et al. Zeolitic imidazolate framework-based electrochemical biosensor for in vivo electrochemical measurements. Anal. Chem. 85, 7550-7557 (2013).

33. Ma, Y. et al. Heterogeneous nano metal-organic framework fluorescence probe for highly selective and sensitive detection of hydrogen sulfide in living cells. Anal. Chem. 86, 11459-114563 (2014).

34. Mao, J. J. et al. Electrocatalytic four-electron reduction of oxygen with Copper (II)-based metal-organic frameworks. Electrochem. Commun. 19, 29-31 (2012).

35. Ahrenholtz, S. R., Epley, C. C. \& Morris, A. J. Solvothermal preparation of an electrocatalytic metalloporphyrin MOF thin film and its redox hopping charge-transfer mechanism. J. Am. Chem. Soc. 136, 2464-2472 (2014).

36. Ling, P. H., Lei, J. P., Zhang, L. \& Ju, H. X. Porphyrin-encapsulated metal-organic frameworks as mimetic catalysts for electrochemical DNA sensing via allosteric switch of hairpin DNA. Anal. Chem. 87, 3957-3963 (2015).

37. Wang, K. C. et al. A series of highly stable mesoporous metalloporphyrin Fe-MOFs. J. Am. Chem. Soc. 136, 13983-13986 (2014).

38. Lykourinou, V. et al. Immobilization of MP-11 into a mesoporous metal-organic framework, MP-11@mesoMOF: a new platform for enzymatic catalysis. J. Am. Chem. Soc. 133, 10382-10385 (2011). 
39. Chen, Y. et al. Why does enzyme not leach from metal-organic frameworks [MOFs]? unveiling the interactions between an enzyme molecule and a MOF. Inorg. Chem. 53, 10006-10008 (2014).

40. Chen, Y. et al. Size-selective biocatalysis of myoglobin immobilized into a mesoporous metal-organic framework with hierarchical pore sizes. Inorg. Chem. 51, 9156-9158 (2012).

41. Chen, Y. et al. How can proteins enter the interior of a MOF? Investigation of cytochrome $\mathrm{c}$ translocation into a MOF consisting of mesoporous cages with microporous windows. J. Am. Chem. Soc. 134, 13188-13191 (2012).

42. Liu, Z. et al. One-pot synthesis of protein-embedded metal-organic frameworks with enhanced biological activities. Nano Lett. 14, $5761-5765$ (2014).

43. Qin, F.X. et al. Hemin@metal-organic framework with peroxidaselike activity and its application to glucose detection. Catal. Sci. Technol. 3, 2761-2768 (2013).

44. Song, Y. H. et al. A green strategy to prepare metal oxide superstructure from metal-organic frameworks. Sci. Rep. 5, 8401-8409 (2015).

45. Kreno, L. E. et al. SERS of molecules that do not adsorb on Ag surfaces: a metal-organic framework-based functionalization strategy. Analyst 139, 4073-4080 (2014).

46. Lin, L. et al. In situ fabrication of a perfect Pd/ZnO@ZIF-8 core-shell microsphere as an efficient catalyst by a ZnO support-induced ZIF-8 growth strategy. Nanoscale 7, 7615-7623 (2015).

47. Ma, M. Y. et al. Iron-based metal-organic frameworks MIL-88B and $\mathrm{NH}_{2}-\mathrm{MIL}-88 \mathrm{~B}$ : high quality microwave synthesis and solventinduced lattice "breathing". Cryst. Growth Des. 13, 2286-2291 (2013).

48. Guo, Y. J. et al. Hemin - graphene hybrid nanosheets with intrinsic peroxidase-like activity for label-free colorimetric detection of single-nucleotide polymorphism. ACS Nano. 5, 1282-1290 (2011).

49. Li, B. L. et al. Hemin-functionalized $\mathrm{MoS}_{2}$ nanosheets: enhanced peroxidase-like catalytic activity with a steady state in aqueous solution. RSC Adv. 4, 24256-24262 (2014).

50. Deng, S. Y. et al. Electrochemiluminescent quenching of quantum dots for ultrasensitive immunoassay through oxygen reduction catalyzed by nitrogen-doped graphene-supported hemin. Anal. Chem. 85, 5390-5396 (2013).

51. Han, J. et al. Employing aqueous CdTe quantum dots with diversified surface functionalities to discriminate between heme [Fe [II]] and hemin [Fe [III]]. Analyst 138, 3402-3408 (2013).

52. Xie, Y. et al. Carbonization of self-assembled nanoporous hemin with a significantly enhanced activity for the oxygen reduction reaction. Faraday discuss. 176, 393-408 (2014).

53. Song, Y. et al. Direct electrochemistry of Cytochrome c based on poly [diallyldimethylammonium Chloride]-graphene nanosheets/ gold nanoparticles hybrid nanocomposites and its biosensing. Electroanalysis 25, 1400-1409 (2013).

54. Samanta, S., Das, P. K., Chatterjee, S. \& Dey, A. Effect of axial ligands on electronic structure and $\mathrm{O}_{2}$ reduction by iron porphyrin complexes: Towards a quantitative understanding of the "push effect". J. Porphyr. Phthalocya. 19, 92-108 (2015).

\section{Acknowledgements}

This work was financially supported by National Natural Science Foundation of China ( 21465014 and 21465015), Natural Science Foundation of Jiangxi Province (20142BAB203010 and 20143ACB21016), Young Scientist Foundation of Jiangxi Province (20122BCB23011), The Ministry of Education by the Specialized Research Fund for the Doctoral Program of Higher Education (20133604110002), the Ground Plan of Science and Technology Projects of Jiangxi Educational Committee (KJLD14023) and the Open Project Program of Key Laboratory of Functional Small Organic Molecule, Ministry of Education, Jiangxi Normal University (No. KLFS-KF-201410; KLFS-KF-201416).

\section{Author Contributions}

L.W., Y.S. and J.H. wrote the main manuscript text. J.H. and H.Y. performed the experiments and prepared Figures 2-5. Y.Z. and J.Y. prepared Figure 6. L.M. prepared Figure 1. All authors reviewed the manuscript.

\section{Additional Information}

Supplementary information accompanies this paper at http://www.nature.com/srep

Competing financial interests: The authors declare no competing financial interests.

How to cite this article: He, J. et al. Smart Nanocomposites of Cu-Hemin Metal-Organic Frameworks for Electrochemical Glucose Biosensing. Sci. Rep. 6, 36637; doi: 10.1038/srep36637 (2016).

Publisher's note: Springer Nature remains neutral with regard to jurisdictional claims in published maps and institutional affiliations.

(c) (i) This work is licensed under a Creative Commons Attribution 4.0 International License. The images or other third party material in this article are included in the article's Creative Commons license, unless indicated otherwise in the credit line; if the material is not included under the Creative Commons license, users will need to obtain permission from the license holder to reproduce the material. To view a copy of this license, visit http://creativecommons.org/licenses/by/4.0/

(C) The Author(s) 2016 